\title{
Uniform attractors for the non-autonomous suspension bridge equation with time delay
}

Su-ping Wang ${ }^{1}$ and Qiao-zhen Ma ${ }^{1 *}$

"Correspondence:

maqzh@nwnu.edu.cn

${ }^{1}$ College of Mathematics and

Statistics, Northwest Normal

University, Lanzhou, China

\begin{abstract}
In this paper, we investigate the existence of a uniform attractor for the non-autonomous suspension bridge equation with time delay by using the energy function and uniform asymptotic compactness of the process.
\end{abstract}

MSC: 35B25; 37L30; 45K05

Keywords: Non-autonomous suspension bridge equation; Uniformly asymptotically compact; Delay; Uniform attractor

\section{Introduction}

A one-dimensional simply supported beam suspended by hangers was modeled as a suspension bridge in [1] by Lazer and McKenna, which described the vibration of the roadbed in the vertical plane. In [1-3], the authors investigated the existence of periodic solutions, the property of traveling wave solutions, and the numerical mountain pass solutions for the suspension bridge equations. Ma and Zhong [4-6] showed the existence of both weak and strong solutions as well as the global attractors for the singled and coupled autonomous suspension bridge equations, respectively. For the non-autonomous case, $\mathrm{Ma}$ et al. $[7,8]$ studied their uniform attractors and pullback attractors. Existence of global attractors for the suspension bridge equations with nonlinear damping was achieved in [9].

However, the above-mentioned models have a drawback, that is, the torsional oscillations in suspension bridges were not described; see [10], for example, the collapse of Tacoma narrow bridge. In the last several decades, the spectacular collapse of the Tacoma narrow bridge has successfully attracted the attention of many engineers, physicists, and mathematicians. They tried their best to explain such an amazing event, and their explanations were mainly based on the aero elastic effects such as the frequency of the vortex shedding, parametric resonance, and flutter theory, but none of their explanations was universally accepted. In 2014, Arioli and Gazzola [11] gave a new explanation about this phenomenon, i.e., the appearance of torsional oscillations during the collapse of Tacoma narrow bridge led to the internal resonances, due to the bridge structure itself. Successively, Ferrero and Gazzola [12] suggested that one should regard a rectangular plate $\Omega=(0, \pi) \times(-l, l)(l \ll \pi)$ as a model of suspension bridges, and the plate was assumed 
to be hinged on its vertical edges

$$
u(0, y, t)=\partial_{x x} u(0, y, t)=u(\pi, y, t)=\partial_{x x} u(\pi, y, t)=0, \quad y \in(-l, l), t>0,
$$

along with the free horizontal edges

$$
\begin{aligned}
& u_{y y}(x, \pm l, t)+\sigma u_{x x}(x, \pm l, t)=0, \\
& u_{y y y}(x, \pm l, t)+(2-\sigma) u_{x x y}(x, \pm l, t)=0, \quad x \in(0, \pi), t>0,
\end{aligned}
$$

where $0<\sigma<\frac{1}{2}$ is the Poisson ratio. They introduced in [12] the following equation as the model of the nonlinear suspension bridges:

$$
u_{t t}(x, y, t)+\delta u_{t}(x, y, t)+\Delta^{2} u(x, y, t)+f(x, y, u)=g(x, y, t), \quad(x, y) \in \Omega, t>0,
$$

where $u(x, y, t)$ is the vertical displacement of the plate in the downward direction, $u_{t}(x, y, t)$ is a damped term, $\delta$ is a positive constant, $f(x, y, u)$ is a restoring force due to the hangers of the suspension bridge, $g(x, y, t)$ is the external force including both gravity and live as well as dead loads. More works related to the suspension bridge equations can be referred to [13-15]. For instance, Al-Gwaiz et al. [13] studied the bending and stretching energies about the rectangular plate model suggested in [12]. Berchio et al. [14] investigated the structural instability of nonlinear plate modeling suspension bridges. For more details on these suspension bridge equations, we refer to the new book [15] published by Gazzola. Besides, Messaoudi et al. [16] obtained the global attractor of nonlinear suspension bridges equations.

Time delays arise usually in physical, chemical, biological, thermal phenomena and so on. In recent years, the partial differential equations with time delay effects have become an active area of research, see [17-28] and the references therein. For example, in [19], the authors showed that a small delay in a boundary control could turn such a well-behaved hyperbolic system into a wild one, so the delay was a source leading to instability of the system. Nicasise and Pignotti in [20] studied the following wave equation with time delay in an open bounded set $\Omega \subset \mathbb{R}^{n}$ :

$$
u_{t t}(x, t)-\Delta u(x, t)+a_{0} u_{t}(x, t)+a_{1} u_{t}(x, t-\tau)=0, \quad x \in \Omega, t>0,
$$

and they proved, under the assumptions that the weight of the feedback is larger than the weight of the delay $\left(a_{1}<a_{0}\right)$, that the energy is exponentially stable. However, they also produced a sequence of delays, which led to the instability of the corresponding solution. The same results were obtained for the case of boundary delay, see [21]. The time delay in a Timoshenko-type system of the form

$$
\begin{cases}\rho_{2} u_{t t}-K\left(u_{x}+\psi\right)_{x}=0, & \text { in }(0, L) \times(0, \infty), \\ \rho_{2} \psi_{t t}-b \psi_{x x}+K\left(u_{x}+\psi\right)+\mu_{1} \psi_{t}+\mu_{2} \psi_{t}(x, t-\tau)=0, & \text { in }(0, L) \times(0, \infty),\end{cases}
$$

was proposed by Said-Houari and Laskri in [22]. They arrived at an exponential decay result under the assumption $\mu_{2}<\mu_{1}$. The authors in [23] considered the well-posedness and exponential stability of wave equation with a strong damping and a strong time delay. 
Recently, Park [24] considered the suspension bridge equations with time delay in a bounded domain $\Omega \subset \mathbb{R}^{2}$

$$
u_{t t}(x, t)+\Delta^{2} u(x, t)+k u^{+}+a_{0} u_{t}(x, t)+a_{1} u_{t}(x, t-\tau)+f(u)=g(x), \quad x \in \Omega, t>0,
$$

and obtained the existence of the finite dimensional global attractors under the conditions that $0<\left|a_{1}\right|<a_{0}$. Also, we would like to recommend many more literature sources such as [25-28] and the references therein. In this paper, we consider the existence of uniform attractors for the following nonlinear plate modeling suspension bridges with time delay in $\Omega=(0, \pi) \times(-l, l)$ :

$$
\begin{gathered}
\partial_{t t} u+\triangle^{2} u+\gamma_{1} \partial_{t} u+\gamma_{2} \partial_{t} u(x, y, t-h)+f(u(x, y, t))=g(x, y, t), \\
(x, y) \in \Omega, t \geq \tau, \tau \in \mathbb{R},
\end{gathered}
$$

with boundary conditions

$$
\begin{cases}u(0, y, t)=\partial_{x x} u(0, y, t)=u(\pi, y, t)=\partial_{x x} u(\pi, y, t)=0, & y \in(-l, l), t \geq \tau, \\ \partial_{y y} u(x, \pm l, t)+\sigma \partial_{x x} u(x, \pm l, t)=0, & x \in(0, \pi), t \geq \tau, \\ \partial_{y y y} u(x, \pm l, t)+(2-\sigma) \partial_{x x y} u(x, \pm l, t)=0, & x \in(0, \pi), t \geq \tau\end{cases}
$$

and initial conditions

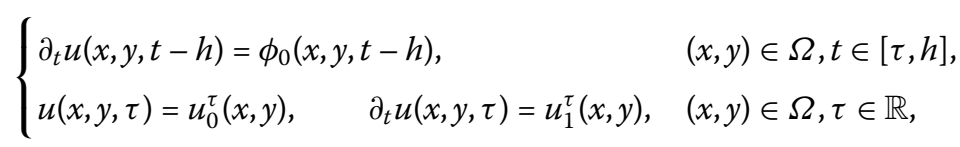

where $\gamma_{1}>0$ is the damped coefficient, $\gamma_{2} \in \mathbb{R}, \partial_{t} u(x, y, t-h)$ is the delay term, and $h>0$ represents the time delay. $u_{0}^{\tau}, u_{1}^{\tau}, \phi_{0}$ are given initial data belonging to suitable space. $g$ and $f$ are external forces. Since we have in mind a long narrow rectangle, that is, $l \ll \pi$, it is reasonable to assume that the forcing term does not depend on $y$, see [12]. So, we now assume that $g(x, y, t)=g(x, t) \in L_{\mathrm{loc}}^{2}\left(\mathbb{R}^{+} ; L^{2}(\Omega)\right)$.

As introduced in [23-25], our equation can be regarded as a Kelvin-Voight linear model for a viscoelastic material in the presence of a time delay response in connection with the stress-strain. Besides, the action of any external force $g(x, y, t)$ on the plate $\Omega$ is transmitted through hangers to the sustaining cables, and this may yield certain delay, see, for example, [25]. It is well known that the presence of delay may lead to instability of the system, and hence it could affect the existence of compact attractors. For these purposes, we will continue to focus on the well-posedness and existence of uniform attractors under the suitable conditions. We extend the results of [25] from the autonomous case to the non-autonomous case.

The paper is organized as follows. In Sect. 2, we present some fundamental and basic results. In Sect. 3, firstly, we show the well-posedness by using maximal monotone operator theory. Secondly, we obtain a uniformly absorbing set. Finally, we establish the existence of a uniform attractor for the process generated by the related problem to (1.2)-(1.4). 


\section{Preliminaries}

We first review some notations about functions spaces and give hypothesis for problem (1.2)-(1.4). From now on, let $c_{i}, i=0,1,2, \ldots$, denote the different positive constants throughout the paper.

As in [12], let us introduce the space

$$
H_{*}^{2}(\Omega)=\left\{w \in H^{2}(\Omega): w(0, y)=w(\pi, y)=0, \forall y \in(-l, l)\right\},
$$

equipped with the inner product and norm

$$
\begin{aligned}
& (u, v)_{H_{*}^{2}}=\int_{\Omega}\left[\Delta u \Delta v+(1-\sigma)\left(2 \partial_{x y} u \partial_{x y} v-\partial_{x x} u \partial_{y y} v-\partial_{y y} u \partial_{x x} v\right)\right] d x d y, \\
& \|u\|_{H_{*}^{2}}=\left[\int_{\Omega}\left[(\Delta u)^{2}+2(1-\sigma)\left(\left(\partial_{x y} u\right)^{2}-\partial_{x x} u \partial_{y y} u\right)\right] d x d y\right]^{\frac{1}{2}} .
\end{aligned}
$$

It was proven that $\|\cdot\|_{H_{*}^{2}}$ is a norm on $H_{*}^{2}$ which is equivalent to the usual $H^{2}(\Omega)$-norm. Moreover, $H_{*}^{2}$ is a Hilbert space when endowed with the scalar product $(\cdot, \cdot)_{H_{*}^{2}}$.

We define the phase space

$$
\mathcal{H}=H_{*}^{2}(\Omega) \times L^{2}(\Omega) \times L^{2}((0,1) \times \Omega)
$$

equipped with the inner product and norm, respectively

$$
\begin{aligned}
& (U, V)_{\mathcal{H}}=(u, \bar{u})_{H_{*}^{2}(\Omega)}+(v, \bar{v})_{L^{2}(\Omega)}+h\left|\gamma_{2}\right|(z, \bar{z})_{L^{2}((0,1) \times \Omega)}, \\
& (U, U)_{\mathcal{H}}=\|u\|_{H_{*}^{2}(\Omega)}^{2}+\|v\|_{L^{2}(\Omega)}^{2}+h\left|\gamma_{2}\right|\|z\|_{L^{2}((0,1) \times \Omega)}^{2},
\end{aligned}
$$

where

$$
U=(u, v, z)^{T}, \quad V=(\bar{u}, \bar{v}, \bar{z})^{T} \in \mathcal{H} .
$$

With regard to problem (1.2)-(1.4), we impose the following assumptions:

(H1) Nonlinear term $f \in C^{1}(\mathbb{R})$ satisfies

$$
\begin{cases}\left|f\left(s_{1}\right)-f\left(s_{2}\right)\right| \leq c_{0}\left(\left|s_{1}\right|^{p}+\left|s_{2}\right|^{p}\right)\left|s_{1}-s_{2}\right|, & \forall s_{1}, s_{2} \in \mathbb{R}, p>0, \\ -c_{1} \leq F(s) \leq s f(s), & \forall s \in \mathbb{R},\end{cases}
$$

where $F(s)=\int_{0}^{s} f(v) d v$.

(H2) The coefficients $\gamma_{1}$ and $\gamma_{2}$ satisfy $0<c\left|\gamma_{2}\right| \leq \gamma_{1}$, where $c$ is a proper positive constant.

As in [20], we introduce the function

$$
z(\rho, x, y, t)=\partial_{t} u(x, y, t-h \rho), \quad \rho \in(0,1),(x, y) \in \Omega, t \geq \tau,
$$

then differentiation with respect to $t$, we can see that

$$
h \partial_{t} z(\rho, x, y, t)+\partial_{\rho} z(\rho, x, y, t)=0, \quad \rho \in(0,1),(x, y) \in \Omega, t \geq \tau .
$$


Thus, problem (1.2)-(1.4) is equivalent to

$$
\begin{cases}\partial_{t t} u+\triangle^{2} u+\gamma_{1} \partial_{t} u+\gamma_{2} z(1)+f(u)=g(x, y, t), & \Omega \times(\tau,+\infty), \\ h \partial_{t} z(\rho, x, y, t)+\partial_{\rho} z(\rho, x, y, t)=0, & (0,1) \times \Omega \times(\tau,+\infty),\end{cases}
$$

with boundary conditions

$$
\begin{cases}u(0, y, t)=\partial_{x x} u(0, y, t)=u(\pi, y, t)=\partial_{x x} u(\pi, y, t)=0, & y \in(-l, l), t \geq \tau \\ \partial_{y y} u(x, \pm l, t)+\sigma \partial_{x x} u(x, \pm l, t)=0, & x \in(0, \pi), t \geq \tau \\ \partial_{y y y} u(x, \pm l, t)+(2-\sigma) \partial_{x x y} u(x, \pm l, t)=0, & x \in(0, \pi), t \geq \tau\end{cases}
$$

and initial conditions

$$
\begin{cases}u(x, y, \tau)=u_{0}^{\tau}(x, y), \quad \partial_{t} u(x, y, \tau)=u_{1}^{\tau}(x, y), & (x, y) \in \Omega, \tau \in \mathbb{R}, \\ z(\rho, x, y, \tau)=\phi_{0}(x, y, \tau-\rho h), & (\rho, x, y) \in(0,1) \times \Omega .\end{cases}
$$

Lemma 2.1 ([12]) Let $u \in H_{*}^{2}(\Omega)$ and suppose $1 \leq p<+\infty$. Then there exists a positive constant $c_{\lambda}=c_{\lambda}(\Omega, p)$ such that

$$
\|u\|_{L^{p}(\Omega)} \leq c_{\lambda}\|u\|_{H_{*}^{2}(\Omega)}
$$

To prove the existence of uniform attractors corresponding to (2.3)-(2.5), we also need the following abstract results.

Let $X$ be a complete metric space and $\Sigma$ be a parameter set.

The operators $\left\{U_{\sigma}(t, \tau)\right\}, \sigma \in \Sigma$ are said to be a family of processes in $X$ with symbol space $\Sigma$ if, for any $\sigma \in \Sigma$,

$$
\begin{aligned}
& U_{\sigma}(t, s) \circ U_{\sigma}(s, \tau)=U_{\sigma}(t, \tau), \quad \forall t \geq s \geq \tau, \tau \in \mathbb{R} \\
& U_{\sigma}(\tau, \tau)=\operatorname{Id} \text { (identity), } \quad \forall \tau \in \mathbb{R} .
\end{aligned}
$$

Let $\{T(r) \mid r \geq 0\}$ be the translation semigroup on $\Sigma$, we say that a family of processes $\left\{U_{\sigma}(t, \tau)\right\}, \sigma \in \Sigma$ satisfies the translation identity if

$$
T(r) \Sigma=\Sigma
$$

and

$$
U_{\sigma}(t+r, \tau+r)=U_{T(r) \sigma}(t, \tau), \quad \forall \sigma \in \Sigma, t \geq \tau, \tau \in \mathbb{R}, r \geq 0
$$

By $\mathcal{B}(E)$ we denote the collection of all bounded subsets of $E$.

Definition $2.2([29,30])$ A bounded set $B_{0} \in \mathcal{B}(E)$ is said to be a bounded uniformly (w.r.t. $\sigma \in \Sigma)$ absorbing set of $\left\{U_{\sigma}(t, \tau)\right\}(\sigma \in \Sigma, t \geq \tau, \tau \in \mathbb{R})$ if, for any $\tau \in \mathbb{R}$ and $B \in \mathcal{B}(E)$, there exists a time $T_{0}=T_{0}(B, \tau) \geq \tau$ such that

$$
\bigcup_{\sigma \in \Sigma} U_{\sigma}(t, \tau) B \subseteq B_{0}
$$

for all $t \geq T_{0}$. 
Definition 2.3 ([30, 31]) Let $E$ be a Banach space and $B \in \mathcal{B}(E), \Sigma$ be a symbol space. We call a function $\psi(\cdot, \cdot ; \cdot, \cdot)$ defined on $(E \times E) \times(\Sigma \times \Sigma)$ to be a contractive function on $B \times B$ if, for any sequence $\left\{x_{n}\right\}_{n=1}^{\infty} \subset B$ and any $\left\{\sigma_{n}\right\} \subset \Sigma$, there is a subsequence $\left\{x_{n_{k}}\right\}_{k=1}^{\infty} \subset\left\{x_{n}\right\}_{n=1}^{\infty}$ and $\left\{\sigma_{n_{k}}\right\}_{k=1}^{\infty} \subset\left\{\sigma_{n}\right\}_{n=1}^{\infty}$ such that

$$
\lim _{k \rightarrow \infty l \rightarrow \infty} \lim _{l \rightarrow \infty}\left(x_{n_{k}}, x_{n_{l}} ; \sigma_{n_{k}}, \sigma_{n_{l}}\right)=0
$$

We denote the set of all contractive functions on $B \times B$ by $\operatorname{Contr}(B, \Sigma)$.

Lemma 2.4 ([32]) Let $\left\{U_{\sigma}(t, \tau)\right\}(\sigma \in \Sigma, t \geq \tau, \tau \in \mathbb{R})$ be a family of processes satisfying the translation identity (2.8)-(2.9) on a Banach space E and having a bounded uniformly (w.r.t. $\sigma \in \Sigma)$ absorbing set $B_{0} \in \mathcal{B}(E)$. Moreover, assume that for any $\varepsilon>0$ there exist $T=T\left(B_{0}, \varepsilon\right)$ and $\psi_{T} \in \operatorname{Contr}\left(B_{0}, \Sigma\right)$ such that

$$
\left\|U_{\sigma_{1}}(T, \tau) x-U_{\sigma_{2}}(T, \tau) y\right\| \leq \varepsilon+\psi\left(x, y ; \sigma_{1}, \sigma_{2}\right), \quad \forall \sigma_{1}, \sigma_{2} \in \Sigma, x, y \in B_{0} .
$$

Then $\left\{U_{\sigma}(t, \tau)\right\}, \sigma \in \Sigma$ is uniformly (w.r.t. $\left.\sigma \in \Sigma\right)$ asymptotically compact in $E$.

Let $X$ be a Banach space with spatial, the space $L_{\text {loc }}^{2}\left(\mathbb{R}^{+} ; X\right)$ denotes all functions with spatial values in Banach space $X$ and time variable locally 2-power integrable in the Bochner sense. $L_{b}^{2}\left(\mathbb{R}^{+} ; X\right)$ is a set of all translation bound functions in $L_{\text {loc }}^{2}\left(\mathbb{R}^{+} ; X\right)$ satisfying

$$
\|\sigma\|_{L_{b}^{2}\left(\mathbb{R}^{+} ; X\right)}=\sup _{t \in \mathbb{R}^{+}} \int_{t}^{t+1}\|\sigma(s)\|_{X}^{2} d s<+\infty
$$

for all $\sigma \in L_{b}^{2}\left(\mathbb{R}^{+} ; X\right)$.

Theorem 2.5 ([30, 32]) Let $E$ be a complete metric space, $\left\{U_{\sigma}(t, \tau)\right\}, \sigma \in \Sigma$ be a family of processes on $E$ satisfying the translation identity (2.8)-(2.9). Then $\left\{U_{\sigma}(t, \tau)\right\}, \sigma \in \Sigma$ has a compactly uniform attractor (w.r.t. $\sigma \in \Sigma$ ) attractor $\mathcal{A}_{\Sigma}$ in $E$ and satisfies

$$
\mathcal{A}_{\Sigma}=\omega_{0, \Sigma}\left(B_{0}\right)=\omega_{\tau, \Sigma}\left(B_{0}\right)=\bigcup_{B \in \mathcal{B}(E)} \omega_{\tau, \Sigma}(B), \quad \forall \tau \in \mathbb{R},
$$

if and only if $\left\{U_{\sigma}(t, \tau)\right\}, \sigma \in \Sigma$

(i) has a bounded uniformly (w.r.t. $\sigma \in \Sigma$ ) absorbing set $B_{0}$;

(ii) is uniformly (w.r.t. $\sigma \in \Sigma$ ) asymptotically compact.

To describe the asymptotic behavior of the solutions to our problem, we set $g_{0} \in$ $L_{b}^{2}\left(\mathbb{R}^{+} ; L^{2}(\Omega)\right)$ and define the symbol space $\mathcal{H}\left(\sigma_{0}\right), \mathcal{H}\left(\sigma_{0}\right)=\mathcal{H}\left(g_{0}\right)=\left[g_{0}(x, s+r) \mid r \in\right.$ $\left.\mathbb{R}^{+}\right]_{L_{\text {loc }}^{2, w}\left(\mathbb{R}^{+} ; L^{2}(\Omega)\right)}$, where [ ] denotes the closure of a set in a topological space $L_{\text {loc }}^{2, w}\left(\mathbb{R}^{+} ; L^{2}(\Omega)\right)$, and $L_{\text {loc }}^{2, w}\left(\mathbb{R}^{+} ; L^{2}(\Omega)\right)$ denotes the space $L_{\text {loc }}^{2, w}\left(\mathbb{R}^{+} ; L^{2}(\Omega)\right)$ endowed with local weak convergence topology. Therefore, for any $g(x, t) \in \mathcal{H}\left(g_{0}\right),(2.3)-(2.5)$ with $g_{0}$ instead of $g$ possesses a corresponding process $\left\{U_{g}(t, \tau)\right\}$ acting on $\mathcal{H}$. The translation semigroup $\{T(r) \mid r \geq 0\}$ satisfies (2.8), (2.9), that is, $T(r) \mathcal{H}\left(g_{0}\right)=\mathcal{H}\left(g_{0}\right)$ and $U_{g}(t+r, \tau+r)=U_{T(r) g(t)}(t, \tau), \forall g \in \mathcal{H}\left(g_{0}\right)$, $t \geq \tau, \tau \in \mathbb{R}, r \geq 0$.

Proposition 2.6 ([29]) If E is a reflexive separable Banach space, then 
(1) for all $g \in \mathcal{H}\left(g_{0}\right),\|g\|_{L_{b}^{2}\left(\mathbb{R}^{+} ; L^{2}(\Omega)\right)} \leq\left\|g_{0}\right\|_{L_{b}^{2}\left(\mathbb{R}^{+} ; L^{2}(\Omega)\right)}$,

(2) the translation group $T(t)$ is weakly continuous on $\mathcal{H}\left(g_{0}\right)$,

(3) $T(t) \mathcal{H}\left(g_{0}\right)=\mathcal{H}\left(g_{0}\right)$ for all $t \in \mathbb{R}$.

\section{Uniform attractors in $\mathcal{H}$}

Firstly, we establish the well-posedness of problem (2.3)-(2.5).

Set $U=(u, v, z)^{T}$, where $v=\partial_{t} u$, initial data $U_{\tau}=\left(u_{0}^{\tau}, u_{1}^{\tau}, \phi_{0}\right)^{T}$, then problem (2.3)-(2.5) is given by

$$
\left\{\begin{array}{l}
U_{t}+A U=F \\
U(\tau)=U_{\tau}
\end{array}\right.
$$

where

$$
A U=\left(\begin{array}{c}
-v \\
\triangle^{2} u+\gamma_{1} v+\gamma_{2} z(1, \cdot) \\
\frac{1}{h} \rho_{z}
\end{array}\right), \quad F(U)=\left(\begin{array}{c}
0 \\
-f(u)+g(t) \\
0
\end{array}\right), \quad U_{\tau}=\left(\begin{array}{l}
u_{0}^{\tau} \\
u_{1}^{\tau} \\
\phi_{0}
\end{array}\right)
$$

The domain of the operator $A$ is defined as follows:

$$
\begin{aligned}
D(A)= & \left\{(u, v, z) \in \mathcal{H} \mid u \in H^{4}(\Omega), v \in H_{*}^{2}(\Omega), z(0, \cdot)=v,\right. \\
& \left.z_{\rho} \in L^{2}((0,1) \times \Omega) \text {, and (3.2) holds }\right\},
\end{aligned}
$$

where (3.2) is the following boundary conditions:

$$
\left\{\begin{array}{l}
\partial_{x x} u(0, y)=\partial_{x x} u(\pi, y)=0 \\
\partial_{y y} u(x, \pm l)+\sigma \partial_{x x} u(x, \pm l)=0 \\
\partial_{y y y} u(x, \pm l)+(2-\sigma) \partial_{x x y} u(x, \pm l)=0
\end{array}\right.
$$

For convenience, we denote $d \theta=d x d y$.

Lemma 3.1 ([33]) Suppose that $0<\left|\gamma_{2}\right| \leq \gamma_{1}$, the operator $A: D(A) \subset \mathcal{H} \rightarrow \mathcal{H}$ is monotone and maximal.

By the semigroup theory, see [33], we have the following result.

Theorem 3.2 Assume that condition (H1) holds and $g \in L_{b}^{2}\left(\mathbb{R}_{\tau} ; L^{2}(\Omega)\right)$. Let $U_{\tau} \in \mathcal{H}$ be given and assume that $\gamma_{1}>\frac{3}{2}\left|\gamma_{2}\right|$, then problem (3.1) has a unique global solution $U=$ $\left(u, \partial_{t} u, \phi\right) \in C\left(\mathbb{R}_{\tau} ; \mathcal{H}\right)$, where $\mathbb{R}_{\tau}=\{t \in \mathbb{R}, t \geq \tau\}$.

Proof Thanks to Lemma 3.1 and $F$ satisfying locally Lipschitz, we can apply the HilleYosida theorem to get the existence of a unique weak local solution for problem (3.1),

$$
U=\left(u, \partial_{t} u, \phi\right) \in C\left(\left[\tau, T_{m}\right], \mathcal{H}\right), \quad \forall T_{m}>0 .
$$

In order to prove that the solution is global and $U \in C([\tau, \infty], \mathcal{H})$, namely we need to prove that $\|U(t)\|_{\mathcal{H}}$ is uniformly bounded with respect to time. 
Multiplying $(2.3)_{1}$ by $\partial_{t} u$ and integrating over $\Omega$, we obtain

$$
\begin{array}{r}
\frac{d}{d t}\left(\frac{1}{2}\left\|\partial_{t} u\right\|_{L^{2}(\Omega)}^{2}+\frac{1}{2}\|u\|_{H_{*}^{2}(\Omega)}^{2}+\int_{\Omega} F(u) d \theta\right) \\
+\gamma_{1}\left\|\partial_{t} u\right\|_{L^{2}(\Omega)}^{2}+\gamma_{2}\left(\partial_{t} u, z(1)\right)=\left(g(t), \partial_{t} u\right)
\end{array}
$$

Multiplying $(2.3)_{2}$ by $\left|\gamma_{2}\right| z$ and integrating over $(0,1) \times \Omega$, we get

$$
h\left|\gamma_{2}\right| \int_{0}^{1} \int_{\Omega} \partial_{t} z z d \theta d \rho+\left|\gamma_{2}\right| \int_{0}^{1} \int_{\Omega} \partial_{\rho} z z d \theta d \rho=0
$$

Thus

$$
\frac{d}{d t}\left(\frac{h\left|\gamma_{2}\right|}{2} \int_{0}^{1} \int_{\Omega}|z|^{2} d \theta d \rho\right)+\frac{\left|\gamma_{2}\right|}{2} \int_{\Omega} \int_{0}^{1} \frac{d}{d \rho}|z|^{2} d \rho d \theta=0
$$

Furthermore, we have

$$
\frac{d}{d t}\left(\frac{h\left|\gamma_{2}\right|}{2} \int_{0}^{1} \int_{\Omega}|z|^{2} d \theta d \rho\right)+\frac{\left|\gamma_{2}\right|}{2} \int_{\Omega}|z(1)|^{2} d \theta-\frac{\left|\gamma_{2}\right|}{2} \int_{\Omega}\left|\partial_{t} u\right|^{2} d \theta=0 .
$$

Set

$$
E_{1}(t)=\frac{1}{2}\left\|\partial_{t} u(t)\right\|_{L^{2}(\Omega)}^{2}+\frac{1}{2}\|u(t)\|_{H_{*}^{2}(\Omega)}^{2}+\int_{\Omega} F(u(t)) d \theta+\frac{1}{2}\|z(t)\|_{L^{2}((0,1) \times \Omega)}^{2} .
$$

Combining (3.3)-(3.5), we get that

$$
\begin{aligned}
\frac{d}{d t} E_{1}(t)= & -\gamma_{1}\left\|\partial_{t} u\right\|_{L^{2}(\Omega)}^{2}-\gamma_{2}\left(\partial_{t} u, z(1)\right)-\frac{\left|\gamma_{2}\right|}{2}\|z(1)\|_{L^{2}(\Omega)}^{2} \\
& +\left|\gamma_{2}\right|\left\|\partial_{t} u\right\|_{L^{2}(\Omega)}^{2}+\frac{1}{2\left|\gamma_{2}\right|}\|g(t)\|_{L^{2}(\Omega)}^{2} .
\end{aligned}
$$

Exploiting Young's inequality, we obtain

$$
-\gamma_{2}\left(\partial_{t} u, z(1)\right) \leq \frac{\left|\gamma_{2}\right|}{2}\|z(1)\|_{L^{2}(\Omega)}^{2}+\frac{\left|\gamma_{2}\right|}{2}\left\|\partial_{t} u\right\|_{L^{2}(\Omega)}^{2}
$$

Substituting (3.8) into (3.7), we find that

$$
\frac{d}{d t} E_{1}(t) \leq-\left(\gamma_{1}-\frac{3}{2}\left|\gamma_{2}\right|\right)\left\|\partial_{t} u\right\|_{L^{2}(\Omega)}^{2}+\frac{1}{2\left|\gamma_{2}\right|}\|g(t)\|_{L^{2}(\Omega)}^{2} .
$$

Integrating (3.9) over $[\tau, t], \gamma_{1}>\frac{3}{2}\left|\gamma_{2}\right|$, we can deduce that

$$
E_{1}(t) \leq E_{1}(\tau)+\frac{1}{2\left|\gamma_{2}\right|} \int_{\tau}^{t}\|g(s)\|_{L^{2}(\Omega)}^{2} d s, \quad \forall t \geq \tau .
$$

By Proposition 2.6, we know that $\|g\|_{L_{b}^{2}\left(\mathbb{R}_{\tau} ; L^{2}(\Omega)\right)}^{2} \leq\left\|g_{0}\right\|_{L_{b}^{2}\left(\mathbb{R}_{\tau} ; L^{2}(\Omega)\right)}^{2}$, one sees that

$$
E_{1}(t) \leq E_{1}(\tau)+\frac{1}{2\left|\gamma_{2}\right|}\left\|g_{0}\right\|_{L_{b}^{2}\left(\mathbb{R}_{\tau} ; L^{2}(\Omega)\right)}^{2}
$$


Applying (H1), $E_{1}(t)$ satisfies

$$
E_{1}(t) \geq \frac{1}{2}\left\|\partial_{t} u(t)\right\|_{L^{2}(\Omega)}^{2}+\frac{1}{2}\|u(t)\|_{H_{*}^{2}(\Omega)}^{2}+\frac{1}{2}\|z(t)\|_{L^{2}((0,1) \times \Omega)}^{2}-c_{1}|\Omega|,
$$

namely

$$
E_{1}(t) \geq c_{2} \|\left(u(t), \partial_{t} u(t), z(t) \|_{\mathcal{H}}^{2}-c_{3}, \quad \forall t \geq \tau\right.
$$

Using (3.10) and (3.11), it yields that

$$
\left\|\left(u(t), \partial_{t} u(t), z(t)\right)\right\|_{\mathcal{H}}^{2} \leq \frac{1}{c_{2}}\left(E_{1}(\tau)+\frac{1}{2\left|\gamma_{2}\right|}\left\|g_{0}\right\|_{L_{b}^{2}\left(\mathbb{R}_{\tau} ; L^{2}(\Omega)\right)}^{2}+c_{3}\right) \leq c_{4}, \quad \forall t \geq \tau .
$$

Owing to Theorem 3.2, we know that (2.3)-(2.5) is well-posed for all $g(s) \in \mathcal{H}\left(g_{0}\right)$ and generates a family of processes $\left\{U_{g}(t, \tau)\right\}, g \in \mathcal{H}\left(g_{0}\right)$ given by $U_{g}(t, \tau) U_{\tau}=U(t)$, where $U(t)$ is the solution of (2.3)-(2.5), and $\left\{U_{g}(t, \tau)\right\}, g \in \mathcal{H}\left(g_{0}\right)$ satisfies (2.7).

In what follows, we denote by $\left\{U_{g}(t, \tau)\right\}, g \in \mathcal{H}\left(g_{0}\right)$ the family of processes generated by (2.3)-(2.5).

Secondly, we prove the existence of a uniformly absorbing set in $\mathcal{H}$. We introduce a Lyapunov functional

$$
L_{1}(t)=M E_{1}(t)+N \Psi_{1}(t)+\Phi_{1}(t)
$$

where $M, N>0$ are constants which will be determined later, and

$$
\Psi_{1}(t)=\left(\partial_{t} u, u\right), \quad \Phi_{1}(t)=h \int_{\Omega} \int_{0}^{1}|z|^{2} e^{-\rho h} d \rho d \theta .
$$

Lemma 3.3 Assume that $N$ is small enough and $M$ is large enough, there exist $\delta_{1}, \delta_{2}>0$ such that

$$
\delta_{1}\left\|\left(u, \partial_{t} u, z\right)\right\|_{\mathcal{H}}^{2}-c_{5} \leq L_{1}(t) \leq \delta_{2}\left\|\left(u, \partial_{t} u, z\right)\right\|_{\mathcal{H}}^{2}+M c_{6} .
$$

Proof On the one hand, by $(\mathrm{H} 1)_{2}$ and the above-mentioned functionals, choosing $M$ large enough and $N$ small enough such that $\frac{M-N}{2}>0, \frac{M-N c_{\lambda}}{2}>0$, we can deduce that

$$
\begin{aligned}
L_{1}(t) \geq & \frac{M}{2}\|u\|_{H_{*}^{2}(\Omega)}^{2}+\frac{M}{2}\left\|\partial_{t} u\right\|_{L^{2}(\Omega)}^{2}+\frac{M h\left|\gamma_{2}\right|}{2} \int_{\Omega} \int_{0}^{1}|z(t)|^{2} e^{-\rho h} d \rho d \theta-c_{1}|\Omega| M \\
& -\frac{N c_{\lambda}}{2}\|u\|_{H_{*}^{2}(\Omega)}^{2}-\frac{N}{2}\left\|\partial_{t} u\right\|_{L^{2}(\Omega)}^{2}+h \int_{\Omega} \int_{0}^{1}|z(t)|^{2} e^{-\rho h} d \rho d \theta \\
\geq & \left(\frac{M-N c_{\lambda}}{2}\right)\|u\|_{H_{*}^{2}(\Omega)}^{2}+\left(\frac{M-N}{2}\right)\left\|\partial_{t} u\right\|_{L^{2}(\Omega)}^{2} \\
& +\left(\frac{\left(M\left|\gamma_{2}\right|+2\right) h}{2}\right) \int_{\Omega} \int_{0}^{1}|z(t)|^{2} e^{-\rho h} d \rho d \theta-c_{1}|\Omega| M \\
\geq & \delta_{1}\left(\|u\|_{H_{*}^{2}(\Omega)}^{2}+\left\|\partial_{t} u\right\|_{L^{2}(\Omega)}^{2}+\|z\|_{L^{2}((0,1) \times \Omega)}^{2}\right)-c_{5} .
\end{aligned}
$$


On the other hand, according to $(\mathrm{H} 1)_{1}$, we have

$$
\begin{aligned}
\int_{\Omega} F(u(t)) d \theta & \leq \int_{\Omega}|u||f(u)| d \theta \leq \int_{\Omega}|u||f(u)-f(0)| d \theta+\int_{\Omega}|u||f(0)| d \theta \\
& \leq c_{0} \int_{\Omega}|u|^{2}|u|^{p} d \theta+\frac{1}{2} \int_{\Omega}|u|^{2} d \theta+\frac{1}{2}|\Omega||f(0)| \\
& \leq c_{0} c_{\lambda}\left(\|u\|_{L^{\infty}(\Omega)}^{p}+1\right)\|u\|_{H_{*}^{2}(\Omega)}^{2}+c_{6} .
\end{aligned}
$$

Exploiting Young's inequality, Sobolev's embedding Theorem, and (3.14), we can get that

$$
\begin{aligned}
L_{1}(t) \leq & \frac{M}{2}\|u\|_{H_{*}^{2}(\Omega)}^{2}+\frac{M}{2}\left\|\partial_{t} u\right\|_{L^{2}(\Omega)}^{2}+\frac{M h\left|\gamma_{2}\right|}{2} \int_{\Omega} \int_{0}^{1}|z(t)|^{2} e^{-\rho h} d \rho d \theta \\
& +M \int_{\Omega} F(u(t)) d \theta+\frac{N}{2}\left\|\partial_{t} u\right\|_{L^{2}(\Omega)}^{2}+\frac{N c_{\lambda}}{2}\|u\|_{H_{*}^{2}(\Omega)}^{2}+h \int_{\Omega} \int_{0}^{1}|z(t)|^{2} e^{-\rho h} d \rho d \theta \\
\leq & \left(\frac{M+N}{2}\right)\left\|\partial_{t} u\right\|_{L^{2}(\Omega)}^{2}+\left(\frac{M+N c_{\lambda}}{2}\right)\|u\|_{H_{*}^{2}(\Omega)}^{2} \\
& +\left(\frac{\left(M\left|\gamma_{2}\right|+2\right) h}{2}\right) \int_{\Omega} \int_{0}^{1}|z(t)|^{2} e^{-\rho h} d \rho d \theta+M \int_{\Omega}|u||f(u)| d \theta \\
\leq & \left(\frac{M+N}{2}\right)\left\|\partial_{t} u\right\|_{L^{2}(\Omega)}^{2}+\left(\frac{M+\left(N+2 M c_{0}\left[\|u\|_{L^{\infty}(\Omega)}^{p}+1\right]\right) c_{\lambda}}{2}\right)\|u\|_{H_{*}^{2}(\Omega)}^{2} \\
& +\left(\frac{\left(M\left|\gamma_{2}\right|+2\right) h}{2}\right) \int_{\Omega} \int_{0}^{1}|z(t)|^{2} e^{-\rho h} d \rho d \theta \\
\leq & \delta_{2}\left(\|u\|_{H_{*}^{2}(\Omega)}^{2}+\left\|\partial_{t} u\right\|_{L^{2}(\Omega)}^{2}+\|z\|_{L^{2}((0,1) \times \Omega)}^{2}\right)+M c_{6} .
\end{aligned}
$$

Lemma 3.4 The function $\Psi_{1}(t)=\left(\partial_{t} u, u\right)$ satisfies

$$
\begin{aligned}
\Psi_{1}^{\prime}(t) \leq & \left(1+\frac{\gamma_{1}}{2 \xi}\right)\left\|\partial_{t} u\right\|_{L^{2}(\Omega)}^{2}+\left[\left(\gamma_{1} \xi+\left|\gamma_{2}\right| \xi+\left|\gamma_{2}\right|\right) \frac{c_{\lambda}}{2}-1\right]\|u\|_{H_{*}^{2}(\Omega)}^{2} \\
& +\frac{\left|\gamma_{2}\right|}{2 \xi}\|z(1)\|_{L^{2}(\Omega)}^{2}+\frac{1}{2\left|\gamma_{2}\right|}\|g(t)\|_{L^{2}(\Omega)}^{2}+c_{1}|\Omega|, \quad \forall \xi>0 .
\end{aligned}
$$

Proof According to $(2.3)_{1}$, we can see that

$$
\begin{aligned}
\Psi_{1}^{\prime}(t)= & \left(\partial_{t t} u, u\right)+\left\|\partial_{t} u\right\|_{L^{2}(\Omega)}^{2} \\
= & \left\|\partial_{t} u\right\|_{L^{2}(\Omega)}^{2}-\|u\|_{H_{*}^{2}(\Omega)}^{2}-\gamma_{1}\left(\partial_{t} u, u\right)-\gamma_{2}(u, z(1)) \\
& -(f(u), u)+(g(t), u)
\end{aligned}
$$

Using Young's inequality, Hölder's inequality, and (2.6), for any $\xi>0$, we can obtain that

$$
\begin{aligned}
& -\gamma_{1}\left(\partial_{t} u, u\right) \leq \frac{\gamma_{1} \xi}{2}\|u\|_{L^{2}(\Omega)}^{2}+\frac{\gamma_{1}}{2 \xi}\left\|\partial_{t} u\right\|_{L^{2}(\Omega)}^{2} \leq c_{\lambda} \frac{\gamma_{1} \xi}{2}\|u\|_{H_{*}^{2}(\Omega)}^{2}+\frac{\gamma_{1}}{2 \xi}\left\|\partial_{t} u\right\|_{L^{2}(\Omega)}^{2}, \\
& -\gamma_{2}(u, z(1)) \leq \frac{\left|\gamma_{2}\right| \xi}{2}\|u\|_{L^{2}(\Omega)}^{2}+\frac{\left|\gamma_{2}\right|}{2 \xi}\|z(1)\|_{L^{2}(\Omega)}^{2} \leq c_{\lambda} \frac{\left|\gamma_{2}\right| \xi}{2}\|u\|_{H_{*}^{2}(\Omega)}^{2}+\frac{\left|\gamma_{2}\right|}{2 \xi}\|z(1)\|_{L^{2}(\Omega)}^{2}, \\
& (g(t), u) \leq \frac{1}{2\left|\gamma_{2}\right|}\|g(t)\|_{L^{2}(\Omega)}^{2}+\frac{c_{\lambda}\left|\gamma_{2}\right|}{2}\|u\|_{H_{*}^{2}(\Omega)}^{2} .
\end{aligned}
$$


By assumption (H1), we have

$$
-(f(u), u) \leq c_{1}|\Omega|
$$

Substituting the above inequalities into (3.16), we get (3.15).

Lemma 3.5 The functional $\Phi_{1}(t)=h \int_{\Omega} \int_{0}^{1}|z|^{2} e^{-\rho h} d \rho d \theta$ satisfies

$$
\Phi_{1}^{\prime}(t) \leq-e^{-h}\left(\|z(1)\|_{L^{2}(\Omega)}^{2}+h \int_{\Omega} \int_{0}^{1}|z(t)|^{2} d \rho d \theta\right)+\left\|\partial_{t} u\right\|_{L^{2}(\Omega)}^{2} .
$$

Proof Direct differentiation $(2.3)_{2}$ leads to

$$
\begin{aligned}
\Phi_{1}^{\prime}(t) & =2 h \int_{\Omega} \int_{0}^{1} \partial_{t} z z e^{-\rho h} d \rho d \theta=-2 \int_{\Omega} \int_{0}^{1} \partial_{\rho} z z e^{-\rho h} d \rho d \theta \\
& =-\int_{\Omega} \int_{0}^{1} \frac{d}{d \rho}\left(|z|^{2} e^{-\rho h}\right) d \rho d \theta-h \int_{0}^{1} \int_{\Omega}|z|^{2} e^{-\rho h} d \theta d \rho \\
& =-e^{-h}\|z(1)\|_{L^{2}(\Omega)}^{2}+\left\|\partial_{t} u\right\|_{L^{2}(\Omega)}^{2}-h \int_{0}^{1} \int_{\Omega}|z|^{2} e^{-\rho h} d \theta d \rho \\
& \leq-e^{-h}\left(\|z(1)\|_{L^{2}(\Omega)}^{2}+h \int_{\Omega} \int_{0}^{1}|z(t)|^{2} d \rho d \theta\right)+\left\|\partial_{t} u\right\|_{L^{2}(\Omega)}^{2} .
\end{aligned}
$$

Theorem 3.6 (Uniformly (w.r.t. $g \in \mathcal{H}\left(g_{0}\right)$ ) absorbing set) Under the assumption of Theorem 3.2, the family of processes $\left\{U_{g}(t, \tau)\right\}, g \in \mathcal{H}\left(g_{0}\right)$ of system (2.3)-(2.5) possesses a bounded uniformly (w.r.t. $\left.g \in \mathcal{H}\left(g_{0}\right)\right)$ absorbing set $B$ in $\mathcal{H}$.

Proof Exploiting (3.9), (3.15), (3.17), Lemma 3.4, and Lemma 3.5, direct computation yields

$$
\begin{aligned}
L_{1}^{\prime}(t)= & M E_{1}^{\prime}(t)+N \Psi_{1}^{\prime}(t)+\Phi_{1}^{\prime}(t) \\
\leq & -N\left(1-\left(\gamma_{1} \xi+\left|\gamma_{2}\right| \xi+\left|\gamma_{2}\right|\right) \frac{c_{\lambda}}{2}\right)\|u\|_{H_{*}^{2}(\Omega)}^{2} \\
& -\left(M\left(\gamma_{1}-\frac{3}{2}\left|\gamma_{2}\right|\right)-N-\frac{\gamma_{1} N}{2 \xi}-1\right)\left\|\partial_{t} u\right\|_{L^{2}(\Omega)}^{2} \\
& -\left(e^{-h}-\frac{N\left|\gamma_{2}\right|}{2 \xi}\right)\|z(1)\|_{L^{2}(\Omega)}^{2}-e^{-h} h\|z\|_{L^{2}((0,1) \times \Omega)}^{2} \\
& +\frac{(M+N)}{2\left|\gamma_{2}\right|}\|g(t)\|_{L^{2}(\Omega)}^{2}+N c_{1}|\Omega| .
\end{aligned}
$$

Choosing $\xi$ and $N$ small enough, $M$ large enough such that

$$
\begin{aligned}
& 1-\left(\gamma_{1} \xi+\left|\gamma_{2}\right| \xi+\left|\gamma_{2}\right|\right) \frac{c_{\lambda}}{2}>0, \quad e^{-h}-\frac{N\left|\gamma_{2}\right|}{2 \xi}>0, \\
& M\left(\gamma_{1}-\frac{3}{2}\left|\gamma_{2}\right|\right)-N-\frac{\gamma_{1} N}{2 \xi}-1>0 .
\end{aligned}
$$


Thus, there exist positive constants $\beta_{1}, \beta_{2}, \beta_{3}, \beta_{4}, \beta_{5}, \beta$ such that

$$
\begin{aligned}
\frac{d L_{1}(t)}{d t} & \leq-\beta_{1}\|u\|_{H_{*}^{2}(\Omega)}^{2}-\beta_{2}\left\|\partial_{t} u\right\|_{L^{2}(\Omega)}^{2}-\beta_{3}\|z\|_{L^{2}((0,1) \times \Omega)}^{2}+\beta_{4}\|g(t)\|_{L^{2}(\Omega)}^{2}+\beta_{5} \\
& \leq-\beta\left(\|u\|_{H_{*}^{2}(\Omega)}^{2}+\left\|\partial_{t} u\right\|_{L^{2}(\Omega)}^{2}+\|z\|_{L^{2}((0,1) \times \Omega)}^{2}\right)+\beta_{4}\|g(t)\|_{L^{2}(\Omega)}^{2}+\beta_{5} .
\end{aligned}
$$

Applying Lemma 3.3, we can get

$$
\frac{d L_{1}(t)}{d t}+v L_{1}(t) \leq c_{7}\|g(t)\|_{L^{2}(\Omega)}^{2}+c_{8}
$$

where $v=\frac{\beta}{\delta_{2}}$. Integrating (3.21) over $[\tau, t]$ and using (3.20), we can see that

$$
\begin{aligned}
L_{1}(t) & \leq L_{1}(\tau) e^{-v(t-\tau)}+c_{7} \int_{\tau}^{t} e^{-v(t-s)}\|g(s)\|_{L^{2}(\Omega)}^{2} d s+c_{8} \int_{\tau}^{t} e^{-v(t-s)} d s \\
& \leq L_{1}(\tau) e^{-v(t-\tau)}+\frac{c_{7}}{1-e^{-\nu}} \sup _{t \geq \tau} \int_{t}^{t+1}\|g(s)\|^{2} d s+c_{9} \\
& \leq L_{1}(\tau) e^{-v(t-\tau)}+\frac{c_{10}}{1-e^{-\nu}}\left\|g_{0}\right\|_{L_{b}^{2}\left(\mathbb{R}_{\tau} ; L^{2}(\Omega)\right)}+c_{9} .
\end{aligned}
$$

Now, for any bounded set $B \subseteq \mathcal{H}$ and for any $\left(u_{0}^{\tau}, u_{1}^{\tau}, \phi_{0}\right) \in B$, there exists a constant $C_{B}>0$ such that $L_{1}(\tau) \leq C_{B}$, then for any $t \geq t_{0}$, we have

$$
L_{1}(t) \leq C_{B} e^{-\nu(t-\tau)}+\frac{c_{10}}{1-e^{-\nu}}\left\|g_{0}\right\|_{L_{b}^{2}\left(\mathbb{R}_{\tau} ; L^{2}(\Omega)\right)}+c_{9}
$$

From (3.23), it is easy to see that

$$
\left\|\left(u(t), \partial_{t} u(t), z(t)\right)\right\|_{\mathcal{H}}^{2} \leq L_{1}(t)=r^{2},
$$

that is, $B=B(0, r)=\left\{\left(u(t), \partial_{t} u(t), z(t)\right) \in \mathcal{H}:\left\|\left(u(t), \partial_{t} u(t), z(t)\right)\right\|_{\mathcal{H}}^{2} \leq r^{2}\right\} \subseteq \mathcal{H}$ is a uniformly absorbing ball for any $g \in \mathcal{H}\left(g_{0}\right)$. The proof is complete.

Next, we will verify that the family of processes $\left\{U_{g}(t, \tau)\right\}, g \in \mathcal{H}\left(g_{0}\right)$ corresponding to (2.3)-(2.5) is uniformly (w.r.t. $\left.g \in \mathcal{H}\left(g_{0}\right)\right)$ asymptotically compact in $\mathcal{H}$.

Theorem 3.7 Assume that condition (H1) holds and $g \in \mathcal{H}\left(g_{0}\right)$. Let $\gamma_{1}>2\left|\gamma_{2}\right|$, then the family of processes $\left\{U_{g}(t, \tau)\right\}, g \in \mathcal{H}\left(g_{0}\right)$ corresponding to (2.3)-(2.5) is uniformly (w.r.t. $g \in$ $\left.\mathcal{H}\left(g_{0}\right)\right)$ asymptotically compact in $\mathcal{H}$.

Proof We consider two symbols $g^{1}, g^{2} \in \mathcal{H}\left(g_{0}\right)$, and let $\left(u^{i}, \partial_{t} u^{i}, z^{i}\right)$ be solutions of (2.3) corresponding to the initial data $\left(u_{0}^{i}, u_{1}^{i}, \phi_{0}^{i}\right)$ and the symbols $g^{1}, g^{2}$, respectively.

Let $u=u^{1}-u^{2}, z=z^{1}-z^{2}, u_{0}^{\tau}=u_{0}^{1 \tau}-u_{0}^{2 \tau}, u_{1}^{\tau}=u_{1}^{1 \tau}-u_{1}^{2 \tau}, \phi_{0}=\phi_{0}^{1}-\phi_{0}^{2}$. Therefore, $(u, z)$ satisfies

$$
\left\{\begin{aligned}
\partial_{t t} u+\triangle^{2} u+\gamma_{1} \partial_{t} u+\gamma_{2} z(1)+f\left(u^{1}\right)-f\left(u^{2}\right) & \\
\quad=g^{1}(t)-g^{2}(t), & \\
h \partial_{t} z(\rho, x, y, t)+\partial_{\rho} z(\rho, x, y, t)=0, & (0,1) \times \Omega \times(\tau,+\infty),
\end{aligned}\right.
$$


with boundary conditions

$$
\begin{cases}u(0, y, t)=\partial_{x x} u(0, y, t)=u(\pi, y, t)=\partial_{x x} u(\pi, y, t)=0, & y \in(-l, l), t \geq \tau \\ \partial_{y y} u(x, \pm l, t)+\sigma \partial_{x x} u(x, \pm l, t)=0, & x \in(0, \pi), t \geq \tau \\ \partial_{y y y} u(x, \pm l, t)+(2-\sigma) \partial_{x x y} u(x, \pm l, t)=0, & x \in(0, \pi), t \geq \tau .\end{cases}
$$

We introduce the function

$$
E_{2}(t)=\frac{1}{2}\left\|\partial_{t} u(t)\right\|_{L^{2}(\Omega)}^{2}+\frac{1}{2}\|u(t)\|_{H_{*}^{2}(\Omega)}^{2}+\frac{1}{2}\|z(t)\|_{L^{2}((0,1) \times \Omega)}^{2},
$$

and

$$
L_{2}(t)=M E_{2}(t)+N \Psi_{2}(t)+\Phi_{2}(t)
$$

where $\Psi_{2}(t)=\left(\partial_{t} u, u\right), \Phi_{2}(t)=h \int_{\Omega} \int_{0}^{1}|z|^{2} e^{-\rho h} d \rho d \theta$.

It is easy to see that $L_{2}(t)$ and $E_{2}(t)$ are equivalent, so there exist two positive constants $\lambda_{1}$ and $\lambda_{2}$ depending on $M, N$ such that

$$
\lambda_{1} E_{2}(t) \leq L_{2}(t) \leq \lambda_{2} E_{2}(t)
$$

where $M>0$ large enough and $N>0$ small enough.

Multiplying (3.24) $)_{1}$ by $\partial_{t} u$ and $(3.24)_{2}$ by $\left|\gamma_{2}\right| z$, then integrating over $\Omega$ and $(0,1) \times \Omega$, respectively, we obtain that

$$
\begin{aligned}
\frac{d}{d t} E_{2}(t) \leq & -\gamma_{1}\left\|\partial_{t} u\right\|_{L^{2}(\Omega)}^{2}+\frac{\left|\gamma_{2}\right|}{2}\|z(1)\|_{L^{2}(\Omega)}^{2}+\frac{\left|\gamma_{2}\right|}{2}\left\|\partial_{t} u\right\|_{L^{2}(\Omega)}^{2} \\
& -\frac{\left|\gamma_{2}\right|}{2}\|z(1)\|_{L^{2}(\Omega)}^{2}+\frac{\left|\gamma_{2}\right|}{2}\left\|\partial_{t} u\right\|_{L^{2}(\Omega)}^{2}-\int_{\Omega}\left(f\left(u^{1}(t)\right)-f\left(u^{2}(t)\right)\right) \partial_{t} u d \theta \\
& +\int_{\Omega}\left(g^{1}(t)-g^{2}(t)\right) \partial_{t} u d \theta \\
\leq & c_{\gamma_{2}} \int_{\Omega}\left|f\left(u^{1}(t)\right)-f\left(u^{2}(t)\right)\right|^{2} d \theta-\left(\gamma_{1}-\frac{3\left|\gamma_{2}\right|}{2}\right)\left\|\partial_{t} u\right\|_{L^{2}(\Omega)}^{2} \\
& +\int_{\Omega}\left(g^{1}(t)-g^{2}(t)\right) \partial_{t} u d \theta .
\end{aligned}
$$

Using $(\mathrm{H} 1)_{1}$, we find that

$$
\begin{aligned}
& \int_{\Omega}\left|f\left(u^{1}(t)\right)-f\left(u^{2}(t)\right)\right|^{2} d \theta \leq c_{0} \int_{\Omega}\left(\left|u^{1}\right|^{2 p}+\left|u^{2}\right|^{2 p}\right)|u|^{2} d \theta \leq c_{B}\|u(\cdot, t)\|_{L^{2}(\Omega)}^{2}, \\
& \int_{\Omega}\left(g^{1}(t)-g^{2}(t)\right) \partial_{t} u d \theta \leq c_{\gamma_{2}}\left\|g^{1}(t)-g^{2}(t)\right\|_{L^{2}(\Omega)}^{2}+\frac{\left|\gamma_{2}\right|}{2}\left\|\partial_{t} u\right\|_{L^{2}(\Omega)}^{2},
\end{aligned}
$$

where $c_{B}, c_{\gamma_{2}}$ are positive constants.

Substituting (3.28), (3.29) into (3.27), we get

$$
\frac{d}{d t} E_{2}(t) \leq c_{B}\|u(\cdot, t)\|_{L^{2}(\Omega)}^{2}-\left(\gamma_{1}-2\left|\gamma_{2}\right|\right)\left\|\partial_{t} u\right\|_{L^{2}(\Omega)}^{2}+c_{\delta_{2}}\left\|g^{1}(t)-g^{2}(t)\right\|_{L^{2}(\Omega)}^{2} .
$$


Similar to the proof of (3.15) and (3.17), we have

$$
\begin{aligned}
\Psi_{2}^{\prime}(t) \leq & \left(1+\frac{\gamma_{1}}{2 \xi}\right)\left\|\partial_{t} u\right\|_{L^{2}(\Omega)}^{2}+\left(\left(\gamma_{1} \xi+\left|\gamma_{2}\right| \xi+3\left|\gamma_{2}\right|\right) \frac{c_{\lambda}}{2}-1\right)\|u\|_{H_{*}^{2}(\Omega)}^{2} \\
& +\frac{\left|\gamma_{2}\right|}{2 \xi}\|z(1)\|_{L^{2}(\Omega)}^{2}+c_{\gamma_{2}}\left\|g^{1}(t)-g^{2}(t)\right\|_{L^{2}(\Omega)}^{2}+c_{B}\|u(\cdot, t)\|_{L^{2}(\Omega)}^{2}
\end{aligned}
$$

and

$$
\Phi_{2}^{\prime}(t) \leq-e^{-h}\left(\|z(1)\|_{L^{2}(\Omega)}^{2}+h \int_{\Omega} \int_{0}^{1}|z(t)|^{2} d \rho d \theta\right)+\left\|\partial_{t} u\right\|_{L^{2}(\Omega)}^{2}
$$

Using (3.30)-(3.32), direct computation

$$
\begin{aligned}
L_{2}^{\prime}(t)= & M E_{2}^{\prime}(t)+N \Psi_{2}^{\prime}(t)+\Phi_{2}^{\prime}(t) \\
\leq & -N\left(1-\left(\gamma_{1} \xi+\left|\gamma_{2}\right| \xi+3\left|\gamma_{2}\right|\right) \frac{c_{\lambda}}{2}\right)\|u\|_{H_{*}^{2}(\Omega)}^{2} \\
& -\left(M\left(\gamma_{1}-2\left|\gamma_{2}\right|\right)-N-\frac{\gamma_{1} N}{2 \xi}-1\right)\left\|\partial_{t} u\right\|_{L^{2}(\Omega)}^{2} \\
& -\left(e^{-h}-\frac{N\left|\gamma_{2}\right|}{2 \xi}\right)\|z(1)\|_{L^{2}(\Omega)}^{2}-e^{-h} h\|z\|_{L^{2}((0,1) \times \Omega)}^{2} \\
& +(M+N) c_{\gamma_{2}}\left\|g^{1}(t)-g^{2}(t)\right\|_{L^{2}(\Omega)}^{2}+(M+N) c_{B}\|u(\cdot, t)\|_{L^{2}(\Omega)}^{2}
\end{aligned}
$$

Choose $\xi$ and $N$ small enough, $M$ large enough such that

$$
\begin{aligned}
& \left.1-\left(\gamma_{1} \xi+\left|\gamma_{2}\right| \xi+3\left|\gamma_{2}\right|\right) \frac{c_{\lambda}}{2}\right)>0, \quad e^{-h}-\frac{N\left|\gamma_{2}\right|}{2 \xi}>0, \\
& M\left(\gamma_{1}-2\left|\gamma_{2}\right|\right)-N-\frac{\gamma_{1} N}{2 \xi}-1>0 .
\end{aligned}
$$

Then there exists $\varpi>0$ such that

$$
\begin{aligned}
\frac{d L_{2}(t)}{d t} \leq & -\varpi E_{2}(t)+(M+N) c_{\gamma_{2}}\left\|g^{1}(t)-g^{2}(t)\right\|_{L^{2}(\Omega)}^{2} \\
& +(M+N) c_{B}\|u(\cdot, t)\|_{L^{2}(\Omega)}^{2} .
\end{aligned}
$$

Exploiting (3.26), from (3.34), we obtain

$$
\frac{d L_{2}(t)}{d t}+\varrho L_{2}(t) \leq(M+N) c_{\gamma_{2}}\left\|g^{1}(t)-g^{2}(t)\right\|_{L^{2}(\Omega)}^{2}+(M+N) c_{c_{B}}\|u(\cdot, t)\|_{L^{2}(\Omega)}^{2}
$$

where $\varrho=\frac{\varpi}{\lambda_{2}}$. Integrating (3.35) over $[\tau, t]$, we can deduce that

$$
\begin{aligned}
L_{2}(t) \leq & L_{2}(\tau) e^{-\varrho(t-\tau)}+(M+N) c_{\gamma_{2}} \int_{\tau}^{t} e^{-\varrho(t-s)}\left\|g^{1}(s)-g^{2}(s)\right\|_{L^{2}(\Omega)}^{2} d s \\
& +(M+N) c_{B} \int_{\tau}^{t} e^{-\varrho(t-s)}\|u(\cdot, s)\|_{L^{2}(\Omega)}^{2} d s .
\end{aligned}
$$


Let $T>\tau$ large enough such that

$$
L_{2}(\tau) e^{-\varrho(T-\tau)} \leq \varepsilon
$$

Together with (3.26), (3.36), and (3.37), it yields

$$
\begin{aligned}
E_{2}(t) & \leq \varepsilon+c_{11} \int_{\tau}^{t}\left\|g^{1}(s)-g^{2}(s)\right\|_{L^{2}(\Omega)}^{2} d s+c_{12} \int_{\tau}^{t}\|u(\cdot, s)\|_{L^{2}(\Omega)}^{2} d s \\
& :=\varepsilon+\psi_{T}\left(\left(u_{0}^{1 \tau}, u_{1}^{1 \tau}, \phi_{0}^{1}\right),\left(u_{0}^{2 \tau}, u_{1}^{2 \tau}, \phi_{0}^{2}\right) ; g^{1}, g^{2}\right) .
\end{aligned}
$$

Subsequently, we will show $\psi_{T}(\cdot, \cdot ;,, \cdot) \in \operatorname{Contr}\left(B, \mathcal{H}\left(g_{0}\right)\right)$ for every fixed $T>\tau$. Because of the existence of a uniformly absorbing set, we can obtain that, for any fixed $T>\tau$ and any bounded set $B$ depending on $T$,

$$
\bigcup_{g \in \mathcal{H}\left(g_{0}\right)} \bigcup_{t \in[\tau, T]} U_{g}(t, \tau) B
$$

is bounded in $\mathcal{H}$. Let the sequence $\left(u_{0 n}^{\tau}, u_{1 n}^{\tau}, \phi_{0 n}\right) \in B, g_{n} \in \mathcal{H}\left(g_{0}\right), n=1,2, \ldots$. Since $B$ is bounded, the corresponding sequence of solutions $\left(u_{n}(t), \partial_{t} u_{n}(t), z_{n}(t)\right)$ of system (2.3)(2.5) is uniformly bounded in $\mathcal{H}$. Hence, $\left(u_{n}, \partial_{t} u_{n}, z_{n}\right)$ is bounded in $C([\tau, T] ; \mathcal{H})$, which implies that $u_{n}$ is bounded in $C\left([\tau, T] ; H^{2}(\Omega)\right)$.

It follows that from the compact embedding of $C\left([\tau, T] ; H^{2}(\Omega)\right) \cap C^{1}\left([\tau, T] ; L^{2}(\Omega)\right)$ into $C\left([\tau, T] ; L^{2}(\Omega)\right)$, there exists a subsequence $\left\{u_{n_{k}}\right\}$ that converges in $C\left([\tau, T] ; L^{2}(\Omega)\right)$, $\forall T>\tau$. So

$$
\lim _{j \rightarrow \infty} \lim _{k \rightarrow \infty} \sup _{t \geq \tau} \int_{\tau}^{t}\left\|u_{n_{j}}(s)-u_{n_{k}}(s)\right\|^{2} d s=0 .
$$

On the other hand, by $g^{n}, g^{m} \in \mathcal{H}\left(g_{0}\right)$, we arrive at

$$
\int_{\tau}^{t}\left\|g^{n}(s)-g^{m}(s)\right\|_{L^{2}(\Omega)}^{2} d s \rightarrow 0, \quad m, n \rightarrow+\infty
$$

Hence, combining with (3.38)-(3.39), we get that $\psi_{T} \in \operatorname{Contr}\left(B, \mathcal{H}\left(g_{0}\right)\right)$, and then this completes the proof of Theorem 3.7 .

Theorem 3.8 (Uniform attractor) Assume that conditions (H1)-(H2) hold and $g \in \mathcal{H}\left(g_{0}\right)$. Then the family of processes $\left\{U_{g}(t, \tau)\right\}, g \in \mathcal{H}\left(g_{0}\right)$ corresponding to (2.3)-(2.5) has a compactly uniform attractor (w.r.t. $\left.g \in \mathcal{H}\left(g_{0}\right)\right) \mathcal{A}_{\Sigma}$ in $\mathcal{H}$.

Proof From Theorem 3.6 and Theorem 3.7 we know that the conditions of Theorem 2.5 are all satisfied.

\footnotetext{
Acknowledgements

The authors express their gratitude to the anonymous referees for their valuable comments and suggestions.

Funding

This work was supported by the National Natural Science Foundation of China (Grant Nos. 11561064, 11761062).
} 


\section{Competing interests}

The authors declare that they have no competing interests.

\section{Authors' contributions}

The authors contributed equally and significantly in writing this paper. All authors read and approved the final manuscript.

\section{Publisher's Note}

Springer Nature remains neutral with regard to jurisdictional claims in published maps and institutional affiliations.

Received: 4 September 2018 Accepted: 10 June 2019 Published online: 24 June 2019

\section{References}

1. Lazer, A.C., McKenna, P.J.: Large scale oscillatory behaviour in loaded asymmetric systems. Ann. Inst. Henri Poincaré, Anal. Non Linéaire 4(3), 243-274 (1987)

2. An, Y:: On the suspension bridge equations and the relevant problems. Doctoral thesis (2001)

3. Humphreys, L.D.: Numerical mountain pass solutions of a suspension bridge equation. Nonlinear Anal. TMA 28(11), 1811-1826 (1997)

4. Ma, Q.Z., Zhong, C.K.: Existence of global attractors for the coupled system of suspension bridge equations. J. Math. Anal. Appl. 308, 365-379 (2005)

5. Ma, Q.Z., Zhong, C.K.: Existence of strong solutions and global attractors for the coupled suspension bridge equations. J. Differ. Equ. 246, 3755-3775 (2009)

6. Zhong, C.K., Ma, Q.Z., Sun, C.Y.: Existence of strong solutions and global attractors for the suspension bridge equations. Nonlinear Anal. 67, 442-454 (2007)

7. Ma, Q.Z., Wang, S.P., Cheng, X.B.: Uniform compact attractors for the coupled suspension bridge equations. Appl. Math. Comput. 217, 6604-6615 (2011)

8. Ma, Q.Z., Wang, B.L.: Existence of pullback attractors for the coupled suspension bridge equations. Electron. J. Differ. Equ. 2011, $16(2011)$

9. Park, J.Y., Kang, J.R.: Global attractors for the suspension bridge equations with nonlinear damping. Q. Appl. Math. 3, 465-475 (2011)

10. McKenna, P.J., Tuama, C.O.: Large torsional oscillations in suspension bridges visited again: vertical forcing creates torsional response. Am. Math. Mon. 108, 738-745 (2001)

11. Arioli, G., Gazzola, F.: A new mathematical explanation of what triggered the catastrophic torsional mode of Tacoma Narrows Bridges collapse. Appl. Math. Model. 39, 901-912 (2015)

12. Ferrero, A., Gazzola, F.: A partially hinged rectangular plate as a model for suspension bridges. Discrete Contin. Dyn. Syst. 35(12), 5879-5908 (2015)

13. Al-Gwaiz, M., Benci, V., Gazzola, F.: Bending and stretching energies in a rectangular plate modeling suspension bridges. Nonlinear Anal. 106, 18-34 (2014)

14. Berchio, E., Ferrero, A., Gazzola, F.: Structural instability of nonlinear plates modeling suspension bridges: mathematical answers to some long-standing questions. Nonlinear Anal., Real World Appl. 28, 91-215 (2016)

15. Gazzola, F.: Mathematical Models for Suspension Bridges: Nonlinear Structural Instability, Modeling, Simulation and Applications, vol. 15. Springer, Berlin (2015)

16. Messaoudi, S.A., Bonfoh, A., Mukiawa, S.E., Enyi, C.D.: The global attractor for a suspension bridge with memory and partially hinged boundary conditions. Z. Angew. Math. Mech. 97(2), 159-172 (2017)

17. Abdallah, C., Dorato, P., Benitez-Read, J., Byrne, R.: Delayed positive feedback can stabilize oscillatory system. In: ACC, San Francisco, pp. 3106-3107 (1993)

18. Suh, I.H., Bien, Z.: Use of time delay action in the controller design. IEEE Trans. Autom. Control 25, 600-603 (1980)

19. Datko, R., Lagnese, J., Polis, M.P.: An example on the effect of time delays in boundary feedback stabilization of wave equations. SIAM J. Control Optim. 24(1), 152-156 (1986)

20. Nicaise, S., Pignotti, C.: Stability and instability results of the wave equation with a delay term in the boundary or internal feedbacks. SIAM J. Control Optim. 45, 1561-1585 (2006)

21. Xu, C.Q., Yung, S.P., Li, L.K.: Stabilization of the wave system with input delay in the boundary control. ESAIM Control Optim. Calc. Var. 12, 770-785 (2006)

22. Said-Houari, B., Laskri, Y.: A stability result for a Timoshenko system with a delay term in the internal feedback. Appl. Math. Comput. 217, 2857-2869 (2010)

23. Messaoudi, S.A., Fareh, A., Doudi, N.: Well posedness and exponential stability in a wave equation with a strong damping and a strong delay. J. Math. Phys. 57, 111501 (2016)

24. Park, S.H.: Long-time behavior for suspension bridge equations with time delay. Z. Angew. Math. Phys. 45, 1-12 (2018)

25. Messaoudi, S.A., Mukiawa, S.E., Cyril, E.D.: Finite dimensional global attractor for a suspension bridge problem with delay. C. R. Acad. Sci. Paris, Ser. I. 354, 808-824 (2016)

26. Caraballo, T., Kloeden, P.E., Real, J.: Pullback and forward attractors for a damped wave equation with delays. Stoch. Dyn. 4(3), 405-423 (2004)

27. Park, S.H.: Long-time dynamics of a von Karman equation with time delay. Appl. Math. Lett. 75, 128-134 (2018)

28. Garcia-Luegngo, J., Marin-Rubio, P.: Reaction-diffusion equations with non-autonomous force in $\mathrm{H}^{-1}$ and delays under measurability conditions on the driving delay term. J. Math. Anal. Appl. 417, 80-95 (2014)

29. Chepyzhov, V.V., Vishik, M.I.: Attractors for Equations of Mathematical Physics. American Mathematical Society Colloquium Publications, vol. 49. Am. Math. Soc., Providence (2002)

30. Feng, B.W., Yang, X.G., Qin, Y.M.: Uniform attractors for a nonautonomous extensible plate equation with a strong damping. Math. Methods Appl. Sci. 40, 3479-3492 (2017) 
31. Khanmamedov, A.K.: Global attractors for a non-autonomous von Karman equations with nonlinear interior dissipation. J. Math. Anal. Appl. 318, 92-101 (2006)

32. Sun, C.Y., Cao, D.M., Duan, J.Q.: Uniform attractors for non-autonomous wave equations with nonlinear damping. SIAM J. Appl. Dyn. Syst. 6, 293-318 (2007)

33. Pazzy, A.: Semigroup of Linear Operators and Application to PDE. Applied Mathematical Sciences, vol. 44. Springer, Berlin (1983)

Submit your manuscript to a SpringerOpen ${ }^{\odot}$ journal and benefit from:

- Convenient online submission

- Rigorous peer review

- Open access: articles freely available online

- High visibility within the field

- Retaining the copyright to your article

Submit your next manuscript at $\boldsymbol{\Delta}$ springeropen.com 